\title{
On the existence of order functions
}

\author{
Ruud Pellikaan*
}

Appeared in:

Journal of Statistical Planning and Inference vol. 94, pp. 287-301, (2001)

\begin{abstract}
The notions of well-behaving sequences and order funtions is fundamental in the elementary treatment of geometric Goppa codes. The existence of order functions is proved with the theory of Gröbner bases.
\end{abstract}

Keywords: Geometric Goppa codes, algebraic geometry codes, Gröbner bases, order function, weight function, discrete valuation.

AMS classification: $94 \mathrm{~B} 25$

\section{Introduction}

In the papers $[5,6,7,11,12]$ a method is given to treat geometric Goppa codes without algebraic geometry, that is to say that the parameters $[n, k, d]$ and the decoding of these codes up to half the (designed) minimum distance can be done without the theory of algebraic curves over finite fields [25], or equivalently the theory of function fields of one variable over finite fields [24], in particular without the theorem of Riemann-Roch. In their treatment the notion of a well-behaving sequence is fundamental. In this paper a method is given to prove the existence of well-behaving sequences or, in the terminology of this paper, the existence of order or weight functions using the theory of Gröbner bases [1, 2].

\footnotetext{
${ }^{*}$ Department of Mathematics and Computing Science, Technical University of Eindhoven , P.O. Box 513, 5600 MB Eindhoven, The Netherlands.
} 
Let $\mathcal{X}$ be an algebraic curve defined over the finite field $\mathbb{F}_{q}$. The genus of the curve $\mathcal{X}$ is denoted by $g$. The function field of rational functions on $\mathcal{X}$ that are defined over $\mathbb{F}_{q}$ is denoted by $\mathbb{F}_{q}(\mathcal{X})$. The principal divisor of a nonzero rational function $f$ is denoted by $(f)$. Let $P_{1}, \ldots, P_{n}$ be $n$ distinct rational points of $\mathcal{X}$. Let $D=P_{1}+\cdots+P_{n}$. Let $G$ be a divisor which has disjoint support with the support of $D$. The vector space $L(G)$ is defined by

$$
L(G)=\left\{f \in \mathbb{F}_{q}(X) \mid f=0 \text { or }(f) \geq-G\right\} .
$$

The index of speciality $i(G)$ of a divisor $G$ is a nonnegative integer which is zero if $\operatorname{deg}(G)>2 g-2$. Then

$$
\operatorname{dim}(L(G))=\operatorname{deg}(G)+1-g+i(G),
$$

by the Theorem of Riemann-Roch. Consider the evaluation map

$$
\text { ev }: L(G) \longrightarrow \mathbb{F}_{q}^{n}
$$

defined by ev(f) $=\left(f\left(P_{1}\right), \ldots, f\left(P_{n}\right)\right)$. The code $C_{L}(D, G)$ is by definition the image of $L(G)$ under this evaluation map. The dual $C_{\Omega}(D, G)$ of the code $C_{L}(D, G)$ can also be defined by a residue map of differential forms. Both these codes have the property that

$$
k+d \geq n+1-g
$$

where $k$ is the dimension and $d$ is the minimum distance of the code.

If $P$ is rational point of $\mathcal{X}$ that is not in the support of $D$, and $G=m P$, then $C_{L}(D, G)$ is called a one point geometric Goppa code. For a fixed $P$ two sequences of codes $\left(C_{L}(D, m P) \mid m \in \mathbb{N}_{0}\right)$ and $\left(C_{\Omega}(D, m P) \mid m \in \mathbb{N}_{0}\right)$ are obtained. The nonnegative integer $m$ is called a (Weierstrass) gap at $P$ if $L(m P)=L((m-1) P)$ and a nongap otherwise. The integer $m$ is a nongap if and only if there exists a rational function $f$ with poles only at $P$ and pole order $m$. So 0 is a nongap and the sum of two nongaps is again a nongap. Hence the nongaps form a (numerical) semigroup. The number of gaps is equal to the genus $g$, and the largest gap is at most $2 g-1$. Let $\left(\rho_{l} \mid l \in \mathbb{N}\right)$ be the sequence of nongaps in increasing order. Then $\rho_{l}=l+g-1$ for all $l>g$.

The rational functions on $\mathcal{X}$ that have only a pole at $P$ form a ring $K_{\infty}(P)$ and the function

$$
\rho: K_{\infty}(P) \rightarrow \mathbb{N}_{0} \cup\{-\infty\}
$$


where $\rho(f)=-v_{P}(f)$ and $v_{P}$ is the discrete valuation at $P$, will be the prime example of a weight function in the terminology of this paper. The map $\rho$ was denoted by deg in [21]. The image of the map $\rho$ is exactly the set of nongaps at $P$ and for every $l$ there exists a function $f_{l} \in K_{\infty}(P)$ such that $\rho\left(f_{l}\right)=\rho_{l}$. In this way a basis $\left(f_{l} \mid l \in \mathbb{N}\right)$ of $K_{\infty}(P)$ over $\mathbb{F}_{q}$ is obtained. Let $L(l)$ be the vector space generated by $f_{1}, \ldots, f_{l}$, or equivalently $L(l)=\left\{f \in K_{\infty}(P) \mid \rho(f) \leq \rho_{l}\right\}$. Let $l(i, j)$ be the smallest positive integer $l$ such that $f_{i} f_{j} \in L(l)$. Then the function $l(i, j)$ is strictly increasing in both arguments, since

$$
\rho_{i}+\rho_{j}=\rho_{l(i, j)}
$$

and $\rho_{i}$ is strictly increasing with $i$.

The same can be done on the level of words in $\mathbb{F}_{q}^{n}$ after applying the evaluation map. Denote the coordinatewise multiplication of $\mathbf{a}$ and $\mathbf{b}$ in $\mathbb{F}_{q}^{n}$ by $\mathbf{a} * \mathbf{b}$. So $\mathbf{a} * \mathbf{b}=\left(a_{1} b_{1}, \ldots, a_{n} b_{n}\right)$. Then $\mathbb{F}_{q}^{n}$ becomes an $\mathbb{F}_{q}$-algebra. The evaluation map can be extended to a map

$$
e v: K_{\infty}(P) \longrightarrow \mathbb{F}_{q}^{n} .
$$

Notice that $e v(f g)=e v(f) * e v(g)$, so this map $e v$ is a morphism of $\mathbb{F}_{q^{-}}$ algebras. Let $\mathbf{f}_{l}=e v\left(f_{l}\right)$. The basis $\mathbf{h}_{1}, \ldots, \mathbf{h}_{n}$ is obtained from this sequence by deleting superfluous elements as follows. The vector $\mathbf{h}_{1}$ is the first nonzero element of the sequence $\left(\mathbf{f}_{l} \mid l \in \mathbb{N}\right)$. Suppose that $\mathbf{h}_{1}, \ldots, \mathbf{h}_{l}$ are defined for $l<n$, then $\mathbf{h}_{l+1}$ is the first element of the sequence $\left(\mathbf{f}_{l} \mid l \in \mathbb{N}\right)$ which is not in the vector space generated by $\mathbf{h}_{1}, \ldots, \mathbf{h}_{l}$. The evaluation map is surjective, so the infinite sequence $\left(\mathbf{f}_{l} \mid l \in \mathbb{N}\right)$ generates $\mathbb{F}_{q}^{n}$ as a vector space. Therefore indeed a basis $\mathbf{h}_{1}, \ldots, \mathbf{h}_{n}$ is obtained.

Let $S(l)$ be the vector space generated by $\mathbf{h}_{1}, \ldots, \mathbf{h}_{l}$. Let $\phi(i, j)$ be the the smallest positive integer such that $\mathbf{h}_{i} * \mathbf{h}_{j} \in S(l)$. The function $\phi(i, j)$ is strictly increasing for many values but not for all values of $i, j$. Well-behaving sequences in $\mathbb{F}_{q}^{n}$ are introduced in terms of the function $\phi(i, j)$ being increasing for certain values of $i$ and $j[5,6]$. In this paper the distinction between functions and words is emphasized and the notion of well-behaving is studied on the level of functions.

Now a more abstract point of view is taken. Let $R$ be an $\mathbb{F}_{q}$-algebra $R$, that is to say a commutative ring with a unit that has $\mathbb{F}_{q}$ as a unitary subring. Suppose that $R$ has a basis consisting of a well-behaving sequence together 
with a surjective morphism $\varphi: R \rightarrow \mathbb{F}_{q}^{n}$ of $\mathbb{F}_{q}$-algebras. In the following section a sketch will be given how this gives rise to a sequence of codes $C(l)$ and a bound on the minimum distance of these codes following the ideas of $[5,6,7,11,12,13,19,20,22,23]$. This was generalized to a bound on the generalized Hamming weights by [10].

In Section 3 the notion of an order function is defined. It is shown that the $\mathbb{F}$-algebra $R$ has a basis consisting of a well-behaving sequence if and only if $R$ has an order function. If $R$ has an order function, then it is an integral domain. A stronger notion than an order function is a weight function. If $R$ has a weight function, then the values of the weight function form a semigroup, and the bounds on the minimum distance of the codes $C(l)$ can be formulated in terms of parameters of this semigroup. See [13].

What remains is to show the existence of well-behaving sequences in an elementary way. This is done in Sections 4 and 5.

\section{A bound on the minimum distance}

Let $\mathbb{F}$ be a field. Let $R$ be an $\mathbb{F}$-algebra. In this paper it is assumed that

$$
\varphi: R \longrightarrow \mathbb{F}^{n}
$$

is a surjective morphism of $\mathbb{F}$-algebras.

Example 2.1 Affine $\mathbb{F}$-algebras. Let the set $\mathcal{P}$ consist of $n$ distinct points $P_{1}, \ldots, P_{n}$ in $\mathbb{F}^{m}$, the affine space over $\mathbb{F}$ of dimension $m$. Consider the evaluation map

$$
e v_{\mathcal{P}}: \mathbb{F}\left[X_{1}, \ldots, X_{m}\right] \longrightarrow \mathbb{F}^{n},
$$

defined by $e v_{\mathcal{P}}(f)=\left(f\left(P_{1}\right), \ldots, f\left(P_{n}\right)\right)$. This is a morphism of $\mathbb{F}$-algebras from $\mathbb{F}\left[X_{1}, \ldots, X_{m}\right]$ to $\mathbb{F}^{n}$, since $f g\left(P_{i}\right)=f\left(P_{i}\right) g\left(P_{i}\right)$ for all polynomials $f$ and $g$, and all $i$. The map $e v_{\mathcal{P}}$ is surjective $[3,10]$.

Suppose that $I$ is an ideal in the ring $\mathbb{F}\left[X_{1}, \ldots, X_{m}\right]$. Let $\mathcal{P}=\left\{P_{1}, \ldots, P_{n}\right\}$ be in the zeroset of $I$ with coordinates in $\mathbb{F}$. So $f\left(P_{j}\right)=0$ for all $f \in I$ and all $j=1, \ldots, n$. Then the evaluation map induces a well-defined linear map

$$
e v_{\mathcal{P}}: \mathbb{F}\left[X_{1}, \ldots, X_{m}\right] / I \longrightarrow \mathbb{F}^{n},
$$

which is also a surjective morphism of $\mathbb{F}$-algebras. 
Definition 2.2 Let $\left(f_{i} \mid i \in \mathbb{N}\right)$ be a basis of $R$ over $\mathbb{F}$. Let $L(l)$ be the vector space generated by $f_{1}, \ldots, f_{l}$. So for all $f \in R$ there exists a positive integer $l \in \mathbb{N}_{0}$ such that $f \in L(l)$. Let $l(i, j)$ be the smallest nonegative integer $l$ such that $f_{i} f_{j} \in L(l)$.

The sequence $\left(f_{i} \mid i \in \mathbb{N}\right)$ is called well-behaving if $l(i, j)$ is strictly increasing in both arguments, that is to say

$$
l(i, j)<l(i+1, j) \text { for all } i, j \in \mathbb{N},
$$

by symmetry. See $[5,6]$, and $[13,19]$ for the similar notion of an errorcorrecting array.

Remark 2.3 The associativity of the triple product $f_{i} f_{j} f_{k}$ implies the associativity of $l(i, j)$, that is to say $l(i, l(j, k)=l(l(i, j), k)$.

Remark 2.4 If $\left(f_{i} \mid i \in \mathbb{N}\right)$ is a well-behaving sequence, then $l(i, j)$ is not zero for all $i, j \in \mathbb{N}$. Because, if $l(i, j)=0$ for $i>1$, then $0 \leq l(i-$ $1, j)<l(i, j)=0$ which is a contradiction. By symmetry it is not possible that $l(i, j)=0$ for $j>1$. Suppose that $l(1,1)=0$. Then $f_{1} f_{1}=0$, so $f_{1} f_{1} f_{3}=0$. So $l(1, l(1,3))=0$. But if $j=l(1,3)$, then $j>l(1,2)>0$. So $l(1, l(1,3)=l(1, j)>0$, which is a contradiction.

Definition 2.5 Let $\mathbb{F}=\mathbb{F}_{q}$. Let $\mathbf{f}_{i}=\varphi\left(f_{i}\right)$. Define the evaluation code $E(l)$ and its dual $C(l)$ by

$$
\begin{gathered}
E(l)=\varphi(L(l))=\left\langle\mathbf{f}_{1}, \ldots, \mathbf{f}_{l}\right\rangle, \\
C(l)=\left\{\mathbf{c} \in \mathbb{F}^{n} \mid \mathbf{c} \cdot \mathbf{f}_{i}=0 \text { for all } i \leq l\right\} .
\end{gathered}
$$

Definition 2.6 Define

$$
N(l)=\left\{(i, j) \in \mathbb{N}^{2} \mid l(i, j)=l+1\right\} .
$$

Let $\nu(l)$ be the number of elements of $N(l)$.

Proposition 2.7 If $\mathbf{y} \in C(l) \backslash C(l+1)$, then $w t(\mathbf{y}) \geq \nu(l)$.

Proof. See $[5,6,7,10,11,12,13,19,20,22,23]$. 
Definition 2.8

$$
\begin{gathered}
d_{O R D}(l)=\min \left\{\nu\left(l^{\prime}\right) \mid l^{\prime} \geq l\right\}, \\
d_{O R D, \varphi}(l)=\min \left\{\nu\left(l^{\prime}\right) \mid l^{\prime} \geq l, C\left(l^{\prime}\right) \neq C\left(l^{\prime}+1\right)\right\},
\end{gathered}
$$

If $R$ is an affine algebra of the form $\mathbb{F}\left[X_{1}, \ldots, X_{m}\right] / I$ and $\varphi$ is the evaluation map $e v_{\mathcal{P}}$ of the set $\mathcal{P}$ of $n$ points in $\mathbb{F}^{m}$, then $d_{O R D, \varphi}$ is denoted by $d_{O R D, \mathcal{P}}$.

Theorem 2.9 The numbers $d_{O R D}(l)$ and $d_{O R D, \varphi}(l)$ are lower bounds for the minimum distance of $C(l)$ :

$$
d(C(l)) \geq d_{O R D, \varphi}(l) \geq d_{O R D}(l) .
$$

Proof. The theorem is a direct consequence of Definition 2.8 and Proposition 2.7.

Remark 2.10 The notation $d_{O R D}$ refers to the order function associated to the well-behaving sequence as will be done in the next section. This bound was called the Feng-Rao bound in [13] and denoted by $d_{F R}$.

The set $N(l)$ and the numbers $\nu(l)$ and $d_{O R D}$ do not depend on the map $\varphi$, or in case $R$ is an affine algebra and $\varphi$ is the evaluation map $e v_{\mathcal{P}}$, on the choice of the set $\mathcal{P}$.

If $\mathcal{P} \subseteq \mathcal{P}^{\prime}$, then $d_{O R D, \mathcal{P}} \geq d_{O R D, \mathcal{P}^{\prime}}$. So, the number $d_{O R D, \mathcal{P}}$ depends on the choice of the set of points. Hence in many examples an improvement of the order bound $d_{O R D}$ is obtained. In particular for the Reed-Muller codes the bound $d_{O R D}$ is very weak wheras $d_{O R D, \mathcal{P}}$ is tight. See $[10,11,12]$.

Example 2.11 Let $R=\mathbb{F}_{q}[X]$. Let $f_{i}=X^{i-1}$. Then $\left(f_{i} \mid i \in \mathbb{N}\right)$ is a wellbehaving sequence of $R$ and $l(i, j)=i+j-1$. Let $\alpha$ be a primitive element of $\mathbb{F}_{q}$. Let $n=q-1$. Let $\varphi: R \rightarrow \mathbb{F}_{q}^{n}$ be the evaluation map defined by $\varphi(f)=\left(f\left(\alpha^{0}\right), f\left(\alpha^{1}\right), \ldots, f\left(\alpha^{n-1}\right)\right)$. Then $C(l)$ is a Reed-Solomon code. The sequence $\alpha^{0}, \alpha^{1}, \ldots, \alpha^{l-1}$ is a defining set of the cyclic code $C(l)$ and $d_{O R D}(l)=l+1$ is the $\mathrm{BCH}$ bound.

Example 2.12 Let $I$ be the ideal in $\mathbb{F}[X, Y]$ generated by

$$
X^{3}+Y^{2}+Y \text {. }
$$

Let $R=\mathbb{F}[X, Y] /\left(X^{3}+Y^{2}+Y\right)$. Let $f_{1}=1, f_{2 i}=X^{i}$ and $f_{2 i+1}=X^{i-1} Y$ for $i \in \mathbb{N}$. Then $\left(f_{i} \mid i \in \mathbb{N}\right)$ is a well-behaving sequence of $R$, and $l(1,1)=1$, $l(1,2)=l(2,1)=2$ and $l(i, j)=i+j$ for all $i, j>1$. Furthermore $\nu(1)=2$ and $\nu(l)=l$ for all $l>1$. So $d_{O R D}(l)=\nu(l)$. 
Remark 2.13 It is possible to give a version of these ideas on the level of words in $\mathbb{F}_{q}^{n}$ without any reference to the functions. See $[5,6,11,12,13,19$, $20,22,23]$. Let $\mathbf{h}_{1}, \ldots, \mathbf{h}_{n}$ be a basis of $\mathbb{F}_{q}^{n}$. Let $S(l)$ be the vector space generated by $\mathbf{h}_{1}, \ldots, \mathbf{h}_{l}$. Let $C_{r}$ be the dual of the code $S(r)$. Let $\phi(i, j)$ be the the smallest positive integer such that $\mathbf{h}_{i} * \mathbf{h}_{j} \in S(l)$. The pair $(i, j)$ is called well-behaving if $\phi\left(i^{\prime}, j^{\prime}\right)<\phi(i, j)$ for all $i^{\prime}, j^{\prime}$ such that $i^{\prime} \leq i, j^{\prime} \leq j$ and $\left(i^{\prime}, j^{\prime}\right) \neq(i, j)$. Define

$$
N_{W B}(l)=\{(i, j) \mid \phi(i, j)=l+1 \text { and }(i, j) \text { is well-behaving }\} .
$$

Let $\nu_{W B}(l)$ be the number of elements of $N_{W B}(l)$. Define

$$
d_{W B}(r)=\min \left\{\nu_{W B}\left(r^{\prime}\right) \mid r \leq r^{\prime}<n\right\} .
$$

Then $d_{W B}(r)$ is a lower bound on the minimum distance of $C_{r}$.

Let the basis $\mathbf{h}_{1}, \ldots, \mathbf{h}_{n}$ be obtained by deleting superfluous elements of the sequence $\left(\mathbf{f}_{l} \mid l \in \mathbb{N}\right)$, where $\left(f_{l} \mid l \in \mathbb{N}\right)$ is a well-behaving sequence of an $\mathbb{F}_{q}$-algebra $R$ with surjective map $\varphi$ and $\mathbf{f}_{l}=\varphi\left(f_{l}\right)$. If the dimension of $C(l)$ is $k, r=n-k$ and $C(l) \neq C(l+1)$, then $d_{W B}(r) \geq d_{O R D, \varphi}(l)$.

\section{Order, degree and weight functions}

Consider the following definitions from $[10,11,12]$.

Definition 3.1 An order function on an $\mathbb{F}$-algebra $R$ is a function

$$
\rho: R \longrightarrow \mathbb{N}_{0} \cup\{-\infty\},
$$

such that the following conditions hold

(O.0) $\rho(f)=-\infty$ if and only if $f=0$

(O.1) $\rho(\lambda f)=\rho(f)$ for all nonzero $\lambda \in \mathbb{F}$

(O.2) $\rho(f+g) \leq \max \{\rho(f), \rho(g)\}$ and equality holds when $\rho(f)<\rho(g)$.

$(O .3)$ If $\rho(f)<\rho(g)$ and $h \neq 0$, then $\rho(f h)<\rho(g h)$

(O.4) If $\rho(f)=\rho(g)$, then there exists a nonzero $\lambda \in \mathbb{F}$ such that $\rho(f-\lambda g)<\rho(g)$.

for all $f, g, h \in R$. Here $-\infty<n$ for all $n \in \mathbb{N}_{0}$. 
Definition 3.2 Let $R$ be an $\mathbb{F}$-algebra. A weight function on $R$ is an order function on $R$ that satisfies furthermore

$$
(O .5) \quad \rho(f g)=\rho(f)+\rho(g)
$$

for all $f, g \in R$. Here $-\infty+n=-\infty$ for all $n \in \mathbb{N}_{0}$.

If $\rho$ is a weight function and $\rho(f)$ is divisible by an integer $d>1$ for all $f \in R$, then $\rho(f) / d$ is again a weight function. Hence we may assume that the greatest common divisor of the integers $\rho(f)$ with $0 \neq f \in R$ is 1 .

Definition 3.3 A degree function on $R$ is a map that satisfies conditions $(O .0),(O .1),(O .2)$ and $(O .5)$.

It is clear that condition $(O .3)$ is a consequence of $(O .5)$.

Example 3.4 The standard example of an $\mathbb{F}$-algebra $R$ with a degree function $\rho$ is obtained by taking $R=\mathbb{F}\left[X_{1}, \ldots, X_{m}\right]$ and $\rho(f)=\operatorname{deg}(f)$, the degree of $f \in R$. It is a weight function if and only if $m=1$.

Theorem 3.5 Let $R$ be an $\mathbb{F}$-algebra. Let $\left(f_{i} \mid i \in \mathbb{N}\right)$ be a well-behaving sequence of $R$. Let $\left(\rho_{i} \mid i \in \mathbb{N}\right)$ be a strictly increasing sequence of nonnegative integers. Define $\rho(0)=-\infty$, and $\rho(f)=\rho_{l}$ if l is the smallest positive integer such that $f \in L(l)$ for a nonzero $f \in R$. Then $\rho$ is an order function on $R$. If moreover $\rho_{l(i, j)}=\rho_{i}+\rho_{j}$, then $\rho$ is a weight function.

Proof. Conditions $(O .0),(O .1),(O .2)$ and $(O .4)$ are a direct consequence of the definitions.

With every nonzero element $f \in R$ the unique positive integer $\iota(f)$ is associated such that $f \in L(\iota(f))$ and $f \notin L(\iota(f)-1)$. So $\rho(f)=\rho_{\iota(f)}$.

Let $f$ and $g$ be nonzero elements of $R$. Then

$$
f=\sum_{i \leq \iota(f)} \lambda_{i} f_{i}, g=\sum_{j \leq \iota(g)} \nu_{j} f_{j} \text { and } f g=\sum_{l \leq \iota(f g)} \mu_{l} f_{l}
$$

with $\lambda_{\iota(f)} \neq 0, \nu_{\iota(g)} \neq 0$ and $\mu_{\iota(f g)} \neq 0$. There exist $\mu_{i j l} \in \mathbb{F}$ such that

$$
f_{i} f_{j}=\sum_{l \leq l(i, j)} \mu_{i j l} f_{l}
$$


and $\mu_{i j l(i, j)} \neq 0$, by definition of $l(i, j)$. So

$$
\mu_{l}=\sum_{l(i, j)=l} \lambda_{i} \nu_{j} \mu_{i j l}
$$

The function $l(i, j)$ is strictly increasing in both arguments, by assumption. So $l(i, j)<l(\iota(f), \iota(g))$ if $i<\iota(f)$ or $j<\iota(g)$. Furthermore, if $i=\iota(f)$ and $j=\iota(g)$, then

$$
\lambda_{i} \nu_{j} \mu_{i j l(i, j)} \neq 0 .
$$

This element is therefore equal to $\mu_{\iota(f g)}$. So $\iota(f g)=l(\iota(f), \iota(g))$. Hence $\rho(f g)=\rho_{l(\iota(f), \iota(g))}$ and $(O .3)$ holds, since $l(i, j)$ is strictly increasing. Therefore $\rho$ is an order function.

If moreover $\rho_{l(i, j)}=\rho_{i}+\rho_{j}$, then

$$
\rho(f g)=\rho_{\iota(f g)}=\rho_{l(\iota(f), \iota(g))}=\rho_{\iota(f)}+\rho_{\iota(g)}=\rho(f)+\rho(g) .
$$

Therefore $\rho$ is a weight function.

Lemma 3.6 Let $\rho$ be an order function on $R$. Then:

1) If $\rho(f)=\rho(g)$, then $\rho(f h)=\rho(g h)$ for all $h \in R$.

2) $\rho(1) \leq \rho(f)$ for all nonzero elements $f \in R$.

3) $\mathbb{F}=\{f \in R \mid \rho(f) \leq \rho(1)\}$.

4) If $\rho(f)=\rho(g)$, then there exists a unique nonzero $\lambda \in \mathbb{F}$ such that $\rho(f-\lambda g)<\rho(g)$

\section{Proof.}

1) If $\rho(f)=\rho(g)$, then there exists a nonzero $\lambda \in \mathbb{F}$ such that $\rho(f-\lambda g)<$ $\rho(g)$, by $(O .4)$. So $\rho(f h-\lambda g h)<\rho(g h)$, by $(O .3)$. Now $f h=(f h-\lambda g h)+$ $\lambda g h$. Hence $\rho(f h)=\rho(\lambda g h)=\rho(g h)$, by $(O .2)$ and $(O .1)$, respectively.

2) Suppose that $f$ is a nonzero element of $R$ such that $\rho(f)<\rho(1)$. Then $\rho(1)>\rho(f)>\rho\left(f^{2}\right)>\cdots$ is a strictly decreasing sequence, by $(O .3)$, but this contradicts the fact that $\mathbb{N}_{0} \cup\{-\infty\}$ is a well-order. Hence $\rho(1) \leq \rho(f)$ for all nonzero elements $f$ in $R$.

3) It is clear that $\mathbb{F}_{q}$ is a subset of $\{f \in R \mid \rho(f) \leq \rho(1)\}$, by $(O .0)$ and (O.1) If $f$ is nonzero and $\rho(f) \leq \rho(1)$, then $\rho(f)=\rho(1)$, by 1$)$. So there exists a nonzero $\lambda \in \mathbb{F}$ such that $\rho(f-\lambda)<\rho(1)$, by $(O .4)$. Hence $f-\lambda=0$ and $f \in \mathbb{F}$.

4) Let $\rho(f)=\rho(g)$. The existence of $\lambda$ is assured by (O.4). Suppose that $\rho(f-\lambda g)<\rho(f)$ and $\rho(f-\mu g)<\rho(f)$ for nonzero $\lambda, \mu \in \mathbb{F}$. Let 
$u=f-\lambda g$ and $v=f-\mu g$. Then $(\mu-\lambda) f=\mu u-\lambda v$. So $\rho((\mu-\lambda) f) \leq$ $\max \{\rho(u), \rho(v)\}<\rho(f)$ by $(O .2)$. If $\mu \neq \lambda$, then $\rho((\mu-\lambda) f)=\rho(f)$, by $(O .1)$, which is a contradiction. Hence $\mu=\lambda$.

Proposition 3.7 If there exists an order function on $R$, then $R$ is an integral domain.

Proof. Suppose that $f g=0$ for some nonzero $f, g \in R$. We may assume that $\rho(f) \leq \rho(g)$. So $\rho\left(f^{2}\right) \leq \rho(f g)=\rho(0)=-\infty$. So $\rho\left(f^{2}\right)=-\infty$, and $f^{2}=0$. Now $f \neq 0$, hence $\rho(1) \leq \rho(f)$, by Lemma 3.6. So $\rho(f) \leq \rho\left(f^{2}\right)=$ $\rho(0)=-\infty$. Hence $f=0$, which is a contradiction. Therefore $R$ has no zero divisors.

Example 3.8 The $\mathbb{F}$-algebra $R=\mathbb{F}\left[X_{1}, X_{2}\right] /\left(X_{1} X_{2}-1\right)$ is an integral domain. Denote the coset of $X_{i}$ modulo the ideal $\left(X_{1} X_{2}-1\right)$ by $x_{i}$. If $\rho$ is an order function on $R$, then $\rho(1) \leq \rho\left(x_{1}\right)$, so $\rho\left(x_{2}\right) \leq \rho\left(x_{1} x_{2}\right)=\rho(1)$, hence $\rho\left(x_{2}\right)=\rho(1)$ and in the same way we get $\rho\left(x_{1}\right)=\rho(1)$. Therefore $\rho(f) \leq \rho(1)$ for all $f \in R$. Hence $\mathbb{F}=R$ by Lemma 3.6, which is a contradiction since $x_{1} \notin \mathbb{F}$. So not every integral domain has an order function.

Proposition 3.9 Let $R$ be an $\mathbb{F}$ algebra with order function $\rho$. Then there exists a well-behaving sequence $\left(f_{i} \mid i \in \mathbb{N}\right)$ of $R$.

Proof. Let $\left(\rho_{i} \mid i \in \mathbb{N}\right)$ be the increasing sequence of all nonnegative integers that appear as the order $\rho(f)$ of a nonzero element $f \in R$. By definition there exists an $f_{i} \in R$ such that $\rho\left(f_{i}\right)=\rho_{i}$ for all $i \in \mathbb{N}$. So $\rho\left(f_{i}\right)<\rho\left(f_{i+1}\right)$ for all $i$, and for all nonzero $f \in R$ there exists an $i$ with $\rho(f)=\rho\left(f_{i}\right)$, by definition. The fact that $\left\{f_{i} \mid i \in \mathbb{N}\right\}$ is a basis is proved by induction and Lemma 3.6 (4). That the function $l(i, j)$ is strictly increasing is a consequence of $(O .3)$.

Remark 3.10 In a sense the theory of algebraic curves is reversed. In the classical way one first has to show, among other things, that the curve is irreducibile, and than one computes a well-behaving sequence of the $\mathbb{F}$-algebra $K_{\infty}(P)$. In the new approach one starts to show that an $\mathbb{F}$-algebra $R$ has an order function, or equivalently a well-behaving sequence, and one gets as a consequence that $R$ is an integral domain. 


\section{On the existence of order and weight func- tions}

The notion of a well-behaving sequence is well known in the theory of Gröbner bases $[1,2]$.

Definition 4.1 Let $R=\mathbb{F}\left[X_{1}, \ldots, X_{m}\right]$. Suppose that $\prec$ is a total order on the set of monomials in the variables $X_{1}, \ldots, X_{m}$ such that for all monomials $M_{1}, M_{2}$, and $M$ the following holds

(R.1) If $M \neq 1$, then $1 \prec M$,

(R.2) If $M_{1} \prec M_{2}$, then $M M_{1} \prec M M_{2}$.

Then $\prec$ is called a reduction, term or admissible order on the monomials.

The multi-index notation is used for monomials. That means $X^{\alpha}=\prod_{i=1}^{m} X_{i}^{\alpha_{i}}$ if $\alpha=\left(\alpha_{1}, \ldots, \alpha_{m}\right)$. The degree of a monomial and of its exponent is defined by

$$
\operatorname{deg}\left(X^{\alpha}\right)=\operatorname{deg}(\alpha)=\sum_{i=1}^{m} \alpha_{i} .
$$

Giving a reduction order on monomials in $m$ variables is the same as giving a total order on $\mathbb{N}_{0}^{m}$ such that, for all $\alpha_{1}, \alpha_{2}$, and $\alpha$ in $\mathbb{N}_{0}^{m}$, the following holds

(E.1) If $\alpha \neq 0$, then $0 \prec \alpha$,

(E.2) If $\alpha_{1} \prec \alpha_{2}$, then $\alpha+\alpha_{1} \prec \alpha+\alpha_{2}$.

We use $\prec$ both for monomials and exponents.

Example 4.2 The lexicographic order $\prec_{L}$ is defined by

$\alpha \prec_{L} \beta$ if and only if $\alpha_{1}=\beta_{1}, \ldots, \alpha_{l-1}=\beta_{l-1}$ and $\alpha_{l}<\beta_{l}$ for some $l, 1 \leq l \leq m$.

The lexicographic order is a reduction order that is not isomorphic with $\mathbb{N}$ with its ordinary order $<$.

Remark 4.3 Let $\prec$ be a reduction order that is isomorphic with $\mathbb{N}$ with its ordinary order $<$. Then the monomials can be listed by the sequence $\left(M_{l} \mid l \in \mathbb{N}\right)$ such that $M_{l} \prec M_{l+1}$ for all $l$. Furthermore for all $i, j$ there exists an $l(i, j)$ such that $M_{i} M_{j}=M_{l(i, j)}$. The function $l(i, j)$ is strictly increasing, since $\prec$ is a reduction order. So $\left(M_{l} \mid l \in \mathbb{N}\right)$ is a well-behaving sequence of $R=\mathbb{F}\left[X_{1}, \ldots, X_{m}\right]$. Hence $R$ has an order function by Theorem 3.5. 
Example 4.4 Let $\mathbf{w}=\left(w_{1}, \ldots, w_{m}\right)$ be an $m$-tuple of positive integers called weights. The weighted degree of $\alpha \in \mathbb{N}_{0}^{m}$ and the corresponding monomial $X^{\alpha}$ is defined by

$$
\operatorname{wdeg}\left(X^{\alpha}\right)=\operatorname{wdeg}(\alpha)=\sum_{i=1}^{m} \alpha_{i} w_{i},
$$

and of a nonzero polynomial $F=\sum \lambda_{\alpha} X^{\alpha}$ by

$$
\operatorname{wdeg}(F)=\max \left\{\operatorname{wdeg}\left(X^{\alpha}\right) \mid \lambda_{\alpha} \neq 0\right\} .
$$

This gives a degree function wdeg on the ring $\mathbb{F}\left[X_{1}, \ldots, X_{m}\right]$. The weighted graded lexicographic order $\prec_{\mathbf{w}}$ on $\mathbb{N}_{0}^{m}$ is defined by

$\alpha \prec_{\mathbf{w}} \beta$ if and only if $\operatorname{wdeg}(\alpha)<\operatorname{wdeg}(\beta)$ or $\operatorname{wdeg}(\alpha)=\operatorname{wdeg}(\beta)$ and $\alpha \prec_{L} \beta$,

and similarly for the monomials. This is indeed a reduction order that is isomorphic with $\mathbb{N}$. Hence $\mathbb{F}\left[X_{1}, \ldots, X_{m}\right]$ has an order function by Remark 4.3 which will be denoted by $\omega$.

Example 4.5 Let $I$ be the ideal in $\mathbb{F}[X, Y]$ generated by a polynomial of the form

$$
X^{a}+Y^{b}+G(X, Y)
$$

with $\operatorname{deg}(G)<\min \{a, b\}$ and $\operatorname{gcd}(a, b)=1$. So it is of type I according to [5], see also [15]. Let $R=\mathbb{F}[X, Y] / I$. Denote the cosets of $X$ and $Y$ modulo $I$ by $x$ and $y$, respectively. Then $x^{a}=-y^{b}-g(x, y)$. So $x^{a}$ is a linear combination of elements of the form $x^{\alpha} y^{\beta}$ with $0 \leq \alpha<a$, since $\operatorname{deg}(G)<b$. By recursion one shows that the set

$$
\left\{x^{\alpha} y^{\beta} \mid 0 \leq \alpha<a\right\}
$$

is a basis for $R$. Using the properties of order functions one shows that $\rho(x)=b$ and $\rho(y)=a$ if there exists a weight function $\rho$ on $R$ such that $\operatorname{gcd}(\rho(x), \rho(y))=1$.

In the following it is shown that indeed such a weight function exists.

Proposition 4.6 Let $I$ be the ideal in $\mathbb{F}[X, Y]$ generated by a polynomial of the form $X^{a}+Y^{b}+G(X, Y)$ with $\operatorname{deg}(G)<\min \{a, b\}$ and $\operatorname{gcd}(a, b)=1$. Let $R=\mathbb{F}[X, Y] / I$. Then there exists a weight function $\rho$ on $R$. The ring $R$ is an integral domain, $I$ is a prime ideal and $X^{a}+Y^{b}+G(X, Y)$ is absolutely irreducible. 
Proof. See also [5, 15]. A generalization of this proposition will be given in Theorem 5.11 and Proposition 5.12. Consider the total weighted degree lexicographic order $\prec_{\mathbf{w}}$ on the monomials in $X$ and $Y$ with respect to the weights $\operatorname{wdeg}(X)=b$ and $\operatorname{wdeg}(Y)=a$. This weight function is injective on the set $\left\{X^{\alpha} Y^{\beta} \mid 0 \leq \alpha<a\right\}$, since $\operatorname{gcd}(a, b)=1$. Let $f_{1}, f_{2}, \ldots$ be an enumeration of the elements $x^{\alpha} y^{\beta}$ of the basis of $R$, and let $\rho_{1}, \rho_{2}, \ldots$ be an enumeration of the nonnegative integers of the form $\alpha b+\beta a$ with $0 \leq \alpha<a$, in such a way that $\rho_{i}<\rho_{i+1}$ and $f_{i}=x^{\alpha} y^{\beta}$ if $\rho_{i}=\alpha b+\beta a$ and $0 \leq \alpha<a$, for all $i$.

It is proved by induction that $\rho_{l(i, j)}=\rho_{i}+\rho_{j}$. The induction is with respect to the well-order $\prec_{\mathbf{w}}$ on $\mathbb{N}^{2}$. Now $f_{1}=1$ and $\rho_{1}=0$. So $l(1,1)=1$ and the start of the induction is satisfied. Suppose that the claim is proved for all $\left(i^{\prime}, j^{\prime}\right) \prec_{\mathbf{w}}(i, j)$. Let $f_{i}=x^{\alpha} y^{\beta}, \rho_{i}=\alpha b+\beta a$ with $0 \leq \alpha<a$. Let $f_{j}=x^{\gamma} y^{\delta}, \rho_{j}=\gamma b+\delta a$ with $0 \leq \gamma<a$. Then $f_{i} f_{j}=x^{\alpha+\gamma} y^{\beta+\delta}$ and $\rho_{i}+\rho_{j}=(\alpha+\gamma) b+(\beta+\delta) a$.

If $\alpha+\gamma<a$, then $f_{i} f_{j}$ is a basis element. So $f_{l(i, j)}=f_{i} f_{j}$ and $\rho_{l(i, j)}=$ $\rho_{i}+\rho_{j}$

If $\alpha+\gamma \geq a$, then $\alpha+\gamma=a+\epsilon$ with $0 \leq \epsilon<a$. So

$$
\rho_{i}+\rho_{j}=(\alpha+\gamma) b+(\beta+\delta) a=\epsilon b+(b+\beta+\delta) a
$$

and

$$
f_{i} f_{j}=-x^{\epsilon} y^{b+\beta+\delta}-x^{\epsilon} g(x, y) .
$$

The term $x^{\epsilon} y^{b+\beta+\delta}$ is a basis element $f_{l}$. We may assume by induction that $x^{\epsilon} g(x, y) \in L(l-1)$, since $\operatorname{deg}(G)<\min \{a, b\}$. So $l=l(i, j)$ and $\rho_{l}=$ $\epsilon b+(b+\beta+\delta) a=\rho_{i}+\rho_{j}$.

So in both cases $f_{i} f_{j} \in L(l) \backslash L(l-1)$, where $l=l(i, j)$ and $\rho_{l}=\rho_{i}+\rho_{j}$. Therefore $l(i, j)<l(i+1, j)$.

Hence there exists a weight function $\rho$ on $R$ such that $\rho\left(x^{\alpha} y^{\beta}\right)=\alpha b+\beta a$, by Theorem 3.5. So $R$ is an integral domain by Proposition 3.7 and $I$ is a prime ideal. These results still hold after extending the field $\mathbb{F}$ to its algebraic closure. Therefore $X^{a}+Y^{b}+G(X, Y)$ is absolutely irreducible.

\section{Gröbner bases and weight functions}

Definition 5.1 Let $\mathbb{F}$ be a field. Let $R=\mathbb{F}\left[X_{1}, \ldots X_{m}\right]$. The set of monomials in $X_{1}, \ldots X_{m}$ will be denoted by $\mathcal{M}$. Let $\prec$ be a reduction order on $\mathcal{M}$. 
If $F=\sum \lambda_{\alpha} X^{\alpha}$, then $\left\{X^{\alpha} \mid \lambda_{\alpha} \neq 0\right\}$ is called the support of $F$ and is denoted by $\operatorname{supp}(F)$. The support is finite and $\prec$ is a total order, so $\operatorname{supp}(F)$ has a largest element that is called the leading monomial of $F$ and is denoted by $\operatorname{lm}(F)$. If $X^{\alpha}$ is the leading monomial of $F$, then $\lambda_{\alpha} X^{\alpha}$ is called the leading term of $F$ and is denoted by $l t(F)$, and $\lambda_{\alpha}$ is called the leading coefficient of $F$ and is denoted by $l c(F)$. The least common multiple of two monomials $X^{\alpha}$ and $X^{\beta}$ is $l c m\left(X^{\alpha}, X^{\beta}\right)=X^{\gamma}$, where $\gamma_{i}=\max \left\{\alpha_{i}, \beta_{i}\right\}$ for all $i$.

Definition 5.2 The partial order $\leq_{P}$ on $\mathcal{M}$ is defined by $X^{\alpha} \leq_{P} X^{\beta}$ if and only if $\alpha_{i} \leq \beta_{i}$ for all $i=1, \ldots, m$.

Definition 5.3 Let $\mathcal{B}$ be a finite subset of $R$. If $F, G \in R$, then $F$ reduces to $G$ with respect to $\mathcal{B}$ if there exists a monomial $X^{\alpha}$ in the support of $F$ with coefficient $\lambda_{\alpha}$, and an element $B \in \mathcal{B}$ such that $l t(B)=\mu_{\beta} X^{\beta}, \beta \leq_{P} \alpha$ and

$$
G=F-\frac{\lambda_{\alpha}}{\mu_{\beta}} B X^{\alpha-\beta} .
$$

This is denoted by $F \rightarrow_{\mathcal{B}} G$, or $F \rightarrow G$ for short. If $F=G$ or there is a sequence $G_{1}, \ldots, G_{k}$ such that $F=G_{1}, G=G_{k}$ and $G_{i} \rightarrow_{\mathcal{B}} G_{i+1}$ for all $1 \leq i<k$, then this is denoted by $F \rightarrow_{\mathcal{B}}^{\bullet} G$.

The ideal generated by $\mathcal{B}$ is denoted by $(\mathcal{B})$. If $F \rightarrow_{\mathcal{B}}^{\bullet} 0$, then $F \in(\mathcal{B})$. A finite set $\mathcal{B}$ in $R$ is called a Gröbner basis if the converse holds as well; that is to say, if $F \rightarrow_{\mathcal{B}}^{\bullet} 0$ for all $F \in(\mathcal{B})$.

The following theorem characterizes Gröbner bases.

Theorem 5.4 Let $\mathcal{B}$ be a finite set in $R$. Then $\mathcal{B}$ is a Gröbner basis if and only if

$$
\{\operatorname{lm}(F) \mid F \in(\mathcal{B}), F \neq 0\}=\{\operatorname{lm}(B M) \mid B \in \mathcal{B}, B \neq 0, M \in \mathcal{M}\} .
$$

Proof. See $[1,2]$.

Definition 5.5 The footprint or $\Delta$-set of a Gröbner basis $\mathcal{B}$ is defined by

$$
\Delta(\mathcal{B})=\mathcal{M} \backslash\{\operatorname{lm}(B M) \mid B \in \mathcal{B}, B \neq 0, M \in \mathcal{M}\} .
$$

Corollary 5.6 If $\mathcal{B}$ is a Gröbner bais for the ideal $I$ in $R$, then the cosets modulo $I$ of the elements of the footprint $\Delta(\mathcal{B})$ form a basis of $R / I$. 
Definition 5.7 Let $F_{1}$ and $F_{2}$ be nonzero polynomials. Let $M_{i}=\operatorname{lm}\left(F_{i}\right)$ and $\lambda_{i}=l c\left(F_{i}\right)$. Then there exist monomials $N_{1}$ and $N_{2}$ such that $M_{i} N_{i}=$ $\operatorname{lcm}\left(M_{1}, M_{2}\right)$ for $i=1,2$. The $S$-polynomial of $F_{1}$ and $F_{2}$ is defined as

$$
S\left(F_{1}, F_{2}\right)=\lambda_{2} N_{1} F_{1}-\lambda_{1} N_{2} F_{2} .
$$

Another useful characterization of Gröbner bases is given by the following.

Proposition 5.8 Let $\mathcal{B}$ be a finite set in $R$. Then $\mathcal{B}$ is a Gröbner basis if and only if $S\left(B_{1}, B_{2}\right) \rightarrow_{\mathcal{B}}^{\bullet} 0$ for all $B_{1}, B_{2} \in(\mathcal{B})$.

Proof. See $[1,2]$.

Remark 5.9 The theory of Gröbner bases can be developed for any $\mathbb{F}$ algebra with an order function.

Let $\prec_{\mathbf{w}}$ be the weighted graded lexicographic order on $\mathbb{F}\left[X_{1}, \ldots, X_{m}\right]$ with respect to the weights $\left(w_{1}, \ldots, w_{m}\right)$. Let $\omega$ be the associated order function. See Example 4.4.

Lemma 5.10 Let $\prec_{\mathbf{w}}$ be the weighted graded lexicographic order. Let $\mathcal{B}$ be a set of polynomials such that every element of $\mathcal{B}$ has exactly two monomials of highest weighted degree in its support. If $F \rightarrow_{\mathcal{B}}^{\bullet} G$ with respect to $\prec_{\mathbf{w}}$ and $F$ has exactly one monomial of highest weighted degree in its support, then $w \operatorname{deg}(F)=w \operatorname{deg}(G)$ and $G$ has exactly one monomial of highest weighted degree in its support.

Proof. By induction it is enough to show the lemma when $F \rightarrow_{\mathcal{B}} G$ and $F$ has exactly one monomial of highest weighted degree in its support. Let $F=F^{\prime}+\lambda_{\alpha} X^{\alpha}$ for some polynomial $F^{\prime}$ such that $\operatorname{wdeg}\left(F^{\prime}\right)<\operatorname{wdeg}(F)$ and $\lambda_{\alpha} X^{\alpha}$ is the leading term of $F$. Let $F \rightarrow_{\mathcal{B}} G$. Then $G=F-\mu M B$ for some $B \in \mathcal{B}$ and monomial $M$ such that $l t(\mu M B)$ is a nonzero term of $F$. So $\operatorname{wdeg}(M B) \leq \operatorname{wdeg}(F)$.

If wdeg $(M B)<\operatorname{wdeg}(F)$, then $G=\left(F^{\prime}-\mu M B\right)+\lambda_{\alpha} X^{\alpha}$. So the weighted degree of $F^{\prime}-\mu M B$ is strictly smaller than wdeg $(G)$, and $F$ and $G$ both have $X^{\alpha}$ as the unique monomial of highest weighted degree in their support.

If $\mathrm{wdeg}(M B)=\operatorname{wdeg}(F)$, then the assumption is used that $B$ has exactly two monomials of highest weighted degree in its support. So there exist a polynomial $B^{\prime}$, monomials $M_{1}, M_{2}$ and nonzero elements $\mu_{1}, \mu_{2} \in \mathbb{F}_{q}$ such 
that $B=B^{\prime}+\mu_{1} M_{1}+\mu_{2} M_{2}, \operatorname{wdeg}\left(B^{\prime}\right)<\operatorname{wdeg}\left(M_{1}\right)=\operatorname{wdeg}\left(M_{2}\right)$ and $M_{1} \prec_{\mathbf{w}} M_{2}$. Therefore the leading term of $\mu M B$ is $\mu \mu_{2} M M_{2}$ is equal to $\lambda_{\alpha} X^{\alpha}$, since it is the only nonzero term of $F$ of weighted degree wdeg $(M B)$. So $G=\left(F^{\prime}-\mu M B^{\prime}\right)-\mu \mu_{1} M M_{1}$ and the weighted degree of $F^{\prime}-\mu M B^{\prime}$ is strictly smaler than $\operatorname{wdeg}(G)=\operatorname{wdeg}\left(M M_{1}\right)$. Hence $\operatorname{wdeg}(G)=\operatorname{wdeg}(F)$ and $M M_{1}$ is the only monomial of highest weighted degree in the support of $G$.

Theorem 5.11 Let $I$ be an ideal in $\mathbb{F}\left[X_{1}, \ldots, X_{m}\right]$ with Gröbner bases $\mathcal{B}$ with respect to $\prec_{\mathrm{w}}$. Suppose that the elements of the footprint of I have mutually distinct weighted degrees and that every element of $\mathcal{B}$ has exactly two monomials of highest weighted degree in its support. Then there exists a weight function $\rho$ on $R=\mathbb{F}\left[X_{1}, \ldots, X_{m}\right] / I$ with the property that $\rho(f)=$ wdeg $(F)$, where $f$ is the coset of $F$ modulo $I$, for all polynomials $F$ with support in $\Delta(I)$.

Proof. The condition on the footprint implies that there exists a sequence $F_{1}, F_{2}, \ldots$ enumerating the elements of the footprint such that wdeg $\left(F_{i}\right)<$ $\operatorname{wdeg}\left(F_{i+1}\right)$ for all $i$. Let $f_{i}$ be the coset of $F_{l}$ modulo $I$. Then $f_{1}, f_{2}, \ldots$ is a basis of $R$ by Corollary 5.6. Let $\rho_{i}=\operatorname{wdeg}\left(F_{i}\right)$. Then $\rho_{i}<\rho_{i+1}$ for all $i$. The product $f_{i} f_{j}$ can be expressed as

$$
f_{i} f_{j}=\sum_{l \leq l(i, j)} \lambda_{l} f_{l}
$$

with $\lambda_{l} \in \mathbb{F}$ for all $l$ and $\lambda_{l(i, j)} \neq 0$. So $F_{i} F_{j}-\sum_{l \leq l(i, j)} \lambda_{l} F_{l} \in I$. Hence

$$
F_{i} F_{j} \rightarrow_{\mathcal{B}} \sum_{l \leq l(i, j)} \lambda_{l} F_{l}
$$

since $\mathcal{B}$ is a Gröbner bases for $I$. Let $F=F_{i} F_{j}$ and $G=\sum_{l \leq l(i, j)} \lambda_{l} F_{l}$. Now $F$ satisfies the assumption of Lemma 5.10 , since $F$ is a monomial. Hence

$$
\rho_{i}+\rho_{j}=\operatorname{wdeg}\left(F_{i} F_{j}\right)=\operatorname{wdeg}(F)=\operatorname{wdeg}(G)=\operatorname{wdeg}\left(F_{l(i, j)}\right)=\rho_{l(i, j)} .
$$

Theorem 3.5 implies that there exists a weight function $\rho$ on $R$ with the stated property. 
Proposition 5.12 Let $I$ be the ideal in $\mathbb{F}\left[X_{1}, \ldots, X_{m}\right]$ generated by

$$
F_{i}=X_{i}^{a_{i}}+X_{i+1}^{b_{i}}+G_{i} \text { for } i=1, \ldots, m-1,
$$

where $G_{i} \in \mathbb{F}\left[X_{1}, \ldots, X_{i+1}\right]$, wdeg $\left(G_{i}\right)<a_{1} \cdots a_{i} b_{i} \cdots b_{m-1}$ and $\operatorname{gcd}\left(a_{i}, b_{j}\right)=$ 1 for all $i \leq j$. Then the ring $R=\mathbb{F}\left[X_{1}, \ldots, X_{m}\right] / I$ has a weight function $\rho$.

Proof. This is a generalization of Proposition 4.6 and a consequence of Theorem 5.11.

Let $w_{i}=a_{1} \cdots a_{i-1} b_{i} \cdots b_{m-1}$. Let $\mathbf{w}=\left(w_{1}, \ldots, w_{m}\right)$. Let $\prec_{\mathbf{w}}$ be the graded lexicographic order with respect to the weights $\mathbf{w}$. Then

$$
\operatorname{wdeg}\left(X_{i}^{a_{i}}\right)=\operatorname{wdeg}\left(X_{i+1}^{b_{i}}\right)=a_{1} \cdots a_{i} b_{i} \cdots b_{m-1}>\operatorname{wdeg}\left(G_{i}\right) .
$$

So $X_{i}^{a_{i}}$ is the leading monomial of $F_{i}$ and $F_{i}$ has exactly two monomials in its support of the same highest weighted degree.

Let $\mathcal{B}=\left\{F_{1}, \ldots, F_{m-1}\right\}$. Then the footprint of $\mathcal{B}$ is equal to

$$
\left\{X^{\alpha} \mid 0 \leq \alpha_{i}<a_{i} \text { for all } 1 \leq i<m\right\} .
$$

The weighted degree of elments of this footprint are mutually distinct, since $\operatorname{gcd}\left(a_{i}, b_{j}\right)=1$ for all $i \leq j$.

Consider the S-polynomial of $F_{i}$ and $F_{j}$

$$
\begin{gathered}
S\left(F_{i}, F_{j}\right)=X_{j}^{a_{j}} X_{i+1}^{b_{i}}+X_{j}^{a_{j}} G_{i}-X_{i}^{a_{i}} X_{j+1}^{b_{j}}-X_{i}^{a_{i}} G_{j}{\stackrel{\rightarrow}{F_{i}}}^{b_{i}}{ }^{b_{i}}-G_{i} G_{j}-X_{i}^{a_{i}} X_{j+1}^{b_{j}}-X_{i}^{a_{i}} G_{j} \stackrel{\bullet_{F}}{ } 0 .
\end{gathered}
$$

So $S\left(F_{i}, F_{j}\right) \rightarrow_{\mathcal{B}}^{\bullet} 0$ for all $F_{i}, F_{j} \in \mathcal{B}$. Hence $\mathcal{B}$ is a Gröbner basis for $I$ by Proposition 5.8. So the ring $R$ has a weight function by Theorem 5.11.

Remark 5.13 Notice that it is essential that one assumes that the $G_{i}$ do not depend on $X_{j}$ for all $j>i+1$. Take for instance $m=3, a_{1}=3, b_{1}=5$, $a_{2}=5, b_{2}=2$ and $G_{1}=X_{3}^{2}, G_{2}=0$, then $X_{1}^{3} \in I=\left(X_{1}^{3}+X_{2}^{5}+X_{3}^{2}, X_{2}^{5}+X_{3}^{2}\right)$, but $X_{1} \notin I$.

The assumption " $\operatorname{gcd}\left(a_{i}, b_{i}\right)=1$ for all $i "$ as in [4], instead of " $\operatorname{gcd}\left(a_{i}, b_{j}\right)=$ 1 for all $i \leq j$ ", is not enough to guarantee the existence of an order function, and as a consequence that $I$ is a prime ideal. Take for example $m=3$, $a_{1}=b_{2}=2, a_{2}=b_{1}=3$ and $G_{1}=G_{2}=0$, then $I=\left(X_{1}^{2}-X_{2}^{3}, X_{2}^{3}-X_{3}^{2}\right)$ is not prime, since

$$
\left(X_{1}-X_{3}\right)\left(X_{1}+X_{3}\right)=X_{1}^{2}-X_{3}^{2}=\left(X_{1}^{2}-X_{2}^{3}\right)+\left(X_{2}^{3}-X_{3}^{2}\right) \in I,
$$

but $X_{1}-X_{3}$ nor $X_{1}+X_{3}$ is an element of $I$. 
This paper was submitted on August 1996. In the meantime it was found that Proposition 5.11 and its converse were shown by Miura $[16,17,18]$. In Matsumoto-Miura [14] this was done by the same Gröbner bases techniques as in this paper. A generalization of these results were obtained by Geil and the author in $[8,9]$.

\section{References}

[1] B. Buchberger, Ein Algorithmus zum Auffinden der Basiselemente des Restklassenringes nach einem nulldimensionalen Polynomideal, Ph.D. thesis, Univ. of Innsbruck, Austria, 1965.

[2] D. Cox, J. Little and D. O'Shea, Ideals, varieties, and algorithms: an introduction to computational algebraic geometry, Springer-Verlag, Berlin 1992.

[3] P. Delsarte, J.M. Goethals and F.J. MacWilliams, "On generalized Reed-Muller codes and their relatives," Information and Control, vol. 16, pp. 403-442, 1970.

[4] G.-L. Feng and T.R.N. Rao, "A class of algebraic geoemtric codes from curves in high-dimensional projective spaces," in $A A E C C$-10, Lect. Notes in Comp. Sc. vol. 673, pp. 132-146, Springer-Verlag, Berlin 1992.

[5] G.-L. Feng and T.R.N. Rao, "A simple approach for construction of algebraic-geometric codes from affine plane curves," IEEE Trans. Inform. Theory, vol. IT-40, pp. 1003-1012, July 1994.

[6] G.-L. Feng and T.R.N. Rao, "Improved geometric Goppa codes Part I, Basic Theory," IEEE Trans. Inform. Theory, vol. IT-41, pp. 1678-1693, Nov. 1995.

[7] G.-L. Feng, V. Wei, T.R.N. Rao and K.K. Tzeng, "Simplified understanding and efficient decoding of a class of algebraic-geometric codes," IEEE Trans. Inform. Theory, vol. IT-40, pp. 981-1002, July 1994.

[8] O. Geil, Codes based on an $\mathbb{F}_{q}$-algebra, Ph.D. Thesis, Aalborg Univ., Denmark, December 1999. 
[9] O. Geil and R. Pellikaan, "The structure of order domains," submitted to Finite Fields and their Applications.

[10] P. Heijnen and R. Pellikaan, "Geneneralized Hamming weights of $q$-ary Reed-Muller codes," IEEE Trans. Inform. Theory, vol. IT-44, pp. 181196, Jan. 1998.

[11] T. Høholdt, J.H. van Lint and R. Pellikaan, "Order functions and evaluation codes," Proc. AAECC-12, Toulouse 23-27 June, 1997, T. Mora and H. Mattson (Eds.), Lect. Notes Comp. Sc., vol. 1255, pp. 138-150, Springer, Berlin 1997.

[12] T. Høholdt, J.H. van Lint and R. Pellikaan, "Algebraic geometry codes," in Handbook of Coding Theory, (V.S. Pless, W.C. Huffman and R.A. Brualdi Eds.), vol 1, pp. 871-961, Elsevier, Amsterdam 1998.

[13] C. Kirfel and R. Pellikaan, "The minimum distance of codes in an array coming from telescopic semigroups," IEEE Trans. Inform. Theory, vol. IT-41, pp. 1720-1732, Nov. 1995.

[14] R. Matsumoto and S. Miura, "On construction and generalization of algebraic geometry codes," to appear in Proc. Algebraic Geometry, Number Theory, Coding Theory and Cryptography.

[15] S. Miura, "Algebraic geometric codes on certain plane curves," IEICE Trans., vol. J75-A, no. 11, pp. 1735-1745, Nov. 1992.

[16] S. Miura, "Constructive theory of algebraic curves," Proc. 17 th Symp. Inform. Theory and its Applications (Hiroshima, Japan), December 1994, pp. 461-464 (Japanese).

[17] S. Miura, Ph.D. Thesis, Univ. Tokyo, May 1997 (Japanese).

[18] S. Miura, "Linear codes on affine algebraic curves," Trans. IEICE, vol. J81-A, no. 10, pp.1398-1421, 1998 (Japanese).

[19] R. Pellikaan, "On the efficient decoding of algebraic-geometric codes," in Eurocode 92 Udine, CISM Courses and Lectures, vol. 339, pp. 231-253, Springer-Verlag, Wien-New York 1993. 
[20] R. Pellikaan, "The shift bound for cyclic, Reed-Muller and geometric Goppa codes," Proceedings AGCT-4, Luminy 1993, pp. 155-175, Walter de Gruyter, Berlin 1996.

[21] S.C. Porter, B.-Z. Shen and R. Pellikaan, "On decoding geometric Goppa codes using an extra place," IEEE Trans. Inform. Theory, vol. IT-38, pp. 1663-1676, Nov. 1992.

[22] B.-Z. Shen and K.K. Tzeng, "Generation of matrices for determining minimum distance and decoding of algebraic-geometric codes" IEEE Trans. Inform. Theory, vol. IT-41, pp. 1703-1708, Nov. 1995.

[23] B.-Z. Shen and K. K. Tzeng, "A code decomposition approach for decoding cyclic and algebraic-geometric codes," IEEE Trans. Inform. Theory, vol. IT-41, pp. 1969-1987, Nov. 1995.

[24] H. Stichtenoth, Algebraic function fields and codes, Universitext, Springer-Verlag, Berlin 1993.

[25] M.A. Tsfasman and S.G. Vlădut, Algebraic-geometric codes, Mathematics and its Applications vol. 58, Kluwer Acad. Publ., Dordrecht 1991. 